\title{
Investigation of Relationship Between Progress of Creative Activity and Brain Activity
}

\author{
Hiromu Sato ${ }^{\mathrm{a}}$, Yuya Chiba ${ }^{\mathrm{b}}$, Yoshiko Maruyama ${ }^{\mathrm{a}}$, Kenji Moriya ${ }^{\mathrm{a},{ }^{*},}$, Masahiro Nakagawa ${ }^{\mathrm{c}}$ \\ aDepartment of Production Systems Engineering, National Institute of Technology, Hakodate college, \\ 14-1 Tokura, Hakodate City, Hokkaido, 042-8501, Japan \\ ${ }^{\mathrm{b} S}$ Support Center for Engineering Education, National Institute of Technology, Hakodate college, \\ 14-1 Tokura, Hakodate City, Hokkaido, 042-8501, Japan \\ 'Department of Science of Technology Innovation, Nagaoka University of Technology, \\ 1603-1 Kamitomioka, Nagaoka City, Niigata, 940-2188, Japan \\ *Corresponding Author: moriya@hakodate-ct.ac.jp
}

\begin{abstract}
Partaking in creative activities such as composing music, producing movies, pictures, or writing novels are an enjoyable aspect of daily life. In instances where an individual comes up with a good idea and/or is excited during a creative activity, occasionally, high quality work and good progress, can be achieved. It has been suggested that the brain becomes active in these conditions. The present study investigated the relationship between progress during a creative activity and brain activity. The composition of music using an electric guitar was chosen as a representative creative activity. Brain activity was measured during composition using 10-channel wearable optical topography, to identify the specific brain- region (namely, the right- or left- prefrontal cortex) that was activated. We observed that the active region depended on the kind of composition melodious or technical. However, there were no distinctive differences in brain activity during composition according to whether progress was good or poor. These results represent data from only one subject; therefore, further experiments are required to obtain accurate conclusions.
\end{abstract}

Keywords: Near-infrared spectroscopy, brain activity, creativity.

\section{Introduction}

Creative activities as well as the act of being creative are important factors in healing and relaxation during daily life ${ }^{(1,2,3)}$. Moreover, creative activities may can comprise an individual's occupation and thus represent the key aspects of an affluent lifestyle. The present research focused on creative activity, in which the formation of a good idea and the enthusiasm that develops occasionally enables performing high -quality work or obtaining good progress in a project. It has been suggested that the brain becomes active in these conditions ${ }^{(4,5)}$. There are researches which attempted to identify the relationship between inspiration and brain activity using a morphing images or an anagram test and so on $^{(4)}$. However, a research using creative activities which do not have definite answer, in particular, a research using music composition is not conducted significantly. Therefore, we investigated the relationship between work progress, selfevaluation, and brain activity during a creative activity; namely, music composition. Investigating this may provide a foundation for the development of methods to stimulate brain activation for creativity. We speculate that such a method is helpful for artists in instances when they require inspiration.

\section{Experimental Methods}

\subsection{Experimental Procedure}

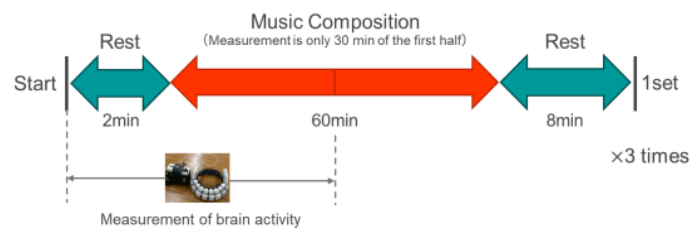

Fig. 1. Timeline illustrating the experimental procedure 
We quantitatively evaluated the brain activity of the subject as they performed a music composition task following a rest task. During the experiment, the subject sat on a chair facing the screen of a personal computer. The subject was instructed to keep their eyes closed during the rest task.

The experimental procedure is shown in Figure 1. The first $2 \mathrm{~min}$ of the procedure comprised a set rest period. The duration of the music composition task was $1 \mathrm{~h}$. During the music composition task, brain activity was measured for 30 min. This time was chosen considering the long duration required for mounting the measurement equipment and the exhaustion that the subject would experience with longer measurement. After the music composition task was completed, the subject rested for $8 \mathrm{~min}$. This sequence was considered as one cycle; the subject repeated the cycle thrice per experiment.

\subsection{Music Composition, Self-Evaluation, and Measurement of Progress}

The music composition task involved the composition of instrumental pieces (vocal composition was not included) of a genre chosen by the subject according to their music preference on a daily basis. Finally, the subject composed four pieces using Digital Audio Workstation software on a personal computer. The subject created the phrases and constitution of the pieces in short sections and either recorded them with an electric guitar or programed drum phrases on the computer. If the subject was satisfied with the creation, they proceeded to the next section; otherwise, they started again. This cycle was repeated until a complete piece was created.

After the completion of composition, the subject's satisfaction with the created piece was evaluated based on the evaluation of the composition by the subject using visual analog scale (VAS) from 0 to 100 points.

Progress was defined using Equation (1), which describes the ratio of the number of bars completed in one experiment to the total number of bars in the piece.

$$
\text { Progress }=\frac{\text { Completed bars in once experiment }}{\text { Entire bars of piece }} \times 100[\%]
$$

\subsection{Measurement and Analysis of Brain Activity}

We quantitatively evaluated brain activity in the prefrontal cortex by measuring changes in the concentration of oxygenated and deoxygenated hemoglobin (oxy-Hb and deoxy-Hb, respectively) using 10-channel wearable optical topography (WOT-100, Hitachi High Technologies Ltd.)
Table. 1. Relationship of brain activity with changes in concentrations of oxygenated and deoxygenated hemoglobin

\begin{tabular}{|c|c|c|}
\hline $\begin{array}{c}\text { Change of } \\
\text { oxy-Hb }\end{array}$ & $\begin{array}{c}\text { Change of } \\
\text { deoxy-Hb }\end{array}$ & Brain Activity \\
\hline & & Active \\
\hline & & Inactive \\
\hline
\end{tabular}

with near-infrared spectroscopy at a sampling frequency of 5 Hz. This method uses near-infrared ray $(700-900 \mathrm{~nm})$ that has high bio-permeability. With the change in the oxidation state of hemoglobin, the intensity of the transmitted nearinfrared ray changes because the absorption spectra of oxy$\mathrm{Hb}$ and deoxy-Hb are different. Therefore, the change in oxidation state can be evaluated from the intensity change of the ray. Typically, an increase in oxy- $\mathrm{Hb}$ and decrease in deoxy-Hb indicates that the prefrontal cortex is active compared with the previous condition and a decrease in oxy$\mathrm{Hb}$ and increase in deoxy-Hb indicates that the prefrontal cortex is inactive (Table 1). We speculated that $\mathrm{Hb}$ concentration would change during the music composition task in line with the above definition of activity and that the degree of change would reflect the progress or selfevaluation of satisfaction.

The measurement regions of the prefrontal cortex corresponding to each measurement channel $(\mathrm{CH})$ are shown in Figure 2. We investigated the following six factors: (1) the activities of the right hemisphere (channels 7-10) and left hemisphere (channels 13-16) in relation to progress and the change in oxy- $\mathrm{Hb}$ concentration in the right and left hemispheres during brain activity; (2) changes in brain activity in relation to the content of music composition, analyzed by subjectively classifying the phrases into "technical" phrases (defined by fast finger movements) or "melodious" phrases (defined by slow finger movements), followed by assessing whether the right or left hemisphere was superior in terms of activity for each phrase (the superior hemisphere was determined based on the change in oxy-Hb concentration; we subtracted mean oxy-Hb concentration change in the left brain from right one. If this difference is a positive value, right -brain superiority was considered because the mean oxy- $\mathrm{Hb}$ concentration change in the right brain is larger than that in the left one, whereas if the difference value is a negative number, left-brain superiority 

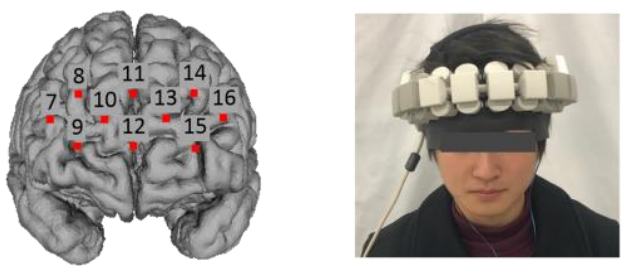

Fig. 2. Regions of the prefrontal cortex (left image) corresponding to the measurement channels of the present study. Measurements of oxygenated and deoxygenated hemoglobin concentrations were conducted using 10-channel wearable optical topography (right image).

was considered); (3) the activity ratio of brain activity [defined by Equation (2)] and its relationship to progress calculated from the brain activity recorded during the 30-min composition time. Active and inactive number in Equation (2) were number of times brain activity was active or inactive during the 30-min composition time.

$$
\text { Activity Ratio }=\frac{\text { Active number }}{\text { Active number }+ \text { Inactive number }} \times 100[\%]
$$

(4) the relationship between mean progress and selfevaluation of satisfaction with completed pieces; (5) the relationship between mean progress and degree of fatigue according to VAS; (6) the relationship between brain activity and the degree of fatigue. To eliminate the influence of sudden change, the first and last 15 seconds of the rest period and the first 30 seconds of the music composition task were excluded from analysis. The $30 \mathrm{~min}$ of the music composition task were separated into 15 -second segments and the mean data was used in analysis.

\section{Results and Discussion}

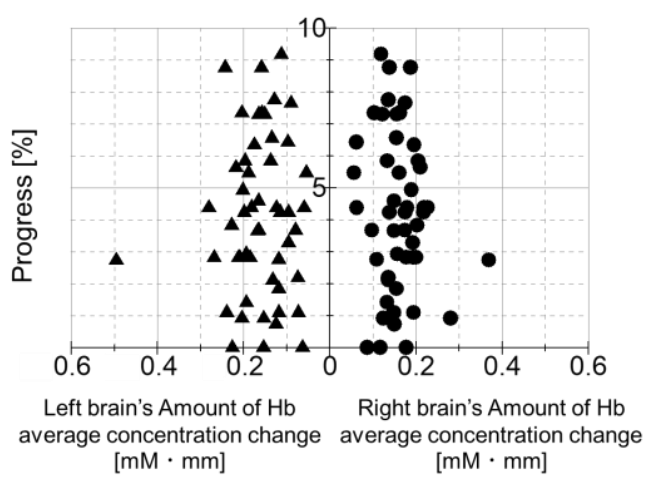

Fig. 3. Relationship between activity of each brain hemisphere and progress during music composition.

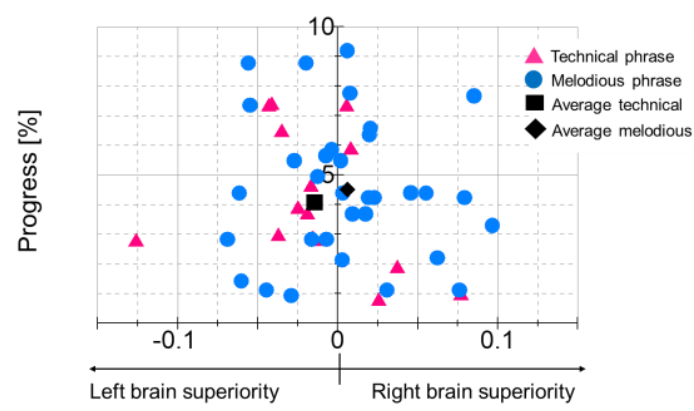

Difference of Oxy-Hb concentration change (Right minus Left) $[\mathrm{mM} \cdot \mathrm{mm}]$

Fig. 4. Correlation between phrase type, brain hemisphere superiority in terms of activity, and progress during music composition

This study included one healthy male who completed cycle of figure 117 times and composed four complete pieces within the same genre. Therefore, we obtained 51 data in total.

\subsection{Relationship between Progress and Right- and Left- Brain Activity}

Figure 3 illustrates the relationship of progress during the music composition task and brain activity. The change in oxy- $\mathrm{Hb}$ concentration was $0.05-0.3 \mathrm{mmol} \cdot \mathrm{mm}$ regardless of progress; therefore, no distinct correlation was observed. We speculated the cause was that there is no change in considering and composing the music using brain even if progress was low.

\subsection{Differences in Brain Hemisphere Superiority According to Phrase Classification}

Figure 4 illustrates the correlation of progress with brain hemisphere superiority for each phrase. No distinct correlation was observed between hemisphere superiority and progress. However, $66.7 \%$ of the technical phrases created were associated with left- brain superiority, whereas $57.6 \%$ of the melodious phrases created were associated

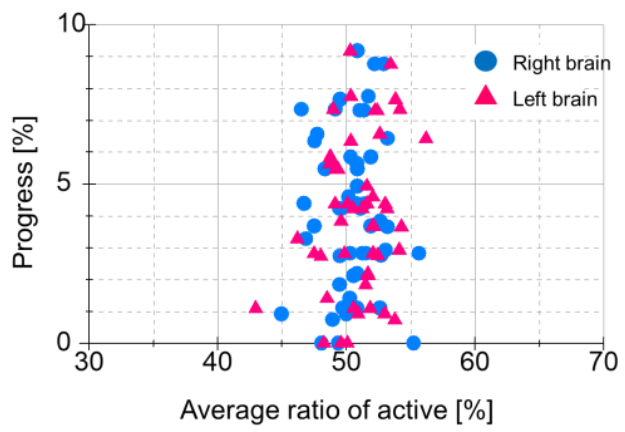

Fig. 5. Correlation between activity ratio and progress during music composition 


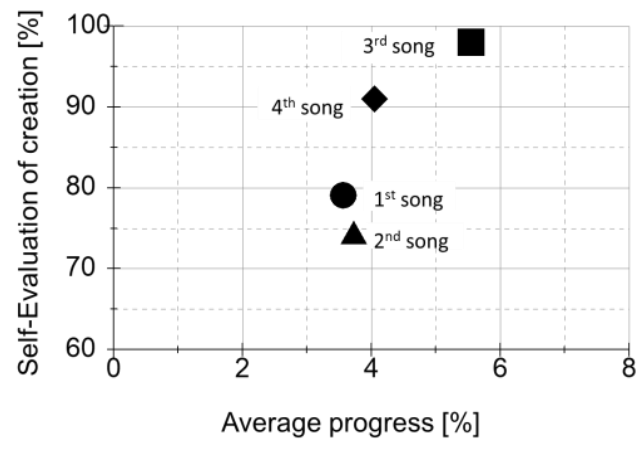

Fig. 6. Relationship between satisfaction (selfevaluation) and mean progress of each composition

with the right-brain superiority. Therefore, there appears to be a relationship between activity of the different brain hemispheres and the type of creative activity or the amount of motion used for playing the instruments ${ }^{(6)}$.

\subsection{Relationship between Progress and Activity Ratio}

Figure 5 presents the results of our analysis of activity ratio in relation to brain activity. The mean activity ratio was approximately $45 \% \sim 55 \%$, regardless of progress; therefore, no distinct correlation was observed. Additionally, it was unclear whether this value was large, because this study included only one subject. The brain of a professional composer may be more active.

\subsection{Relationship between Self-Evaluation and Mean Progress}

Figure 6 shows the relationship between the mean progress of each composition and self-evaluation of satisfaction using VAS. The subject's satisfaction with the composition appeared to be positively correlated with progress. However, the sample size of the present study was small (four compositions), and therefore, more data must be collected and analyzed to confirm this conclusion.

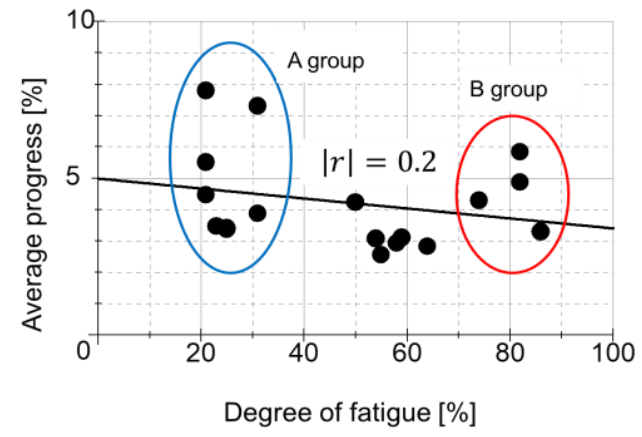

Fig. 7. Correlation of the degree of fatigue with mean progress during music composition

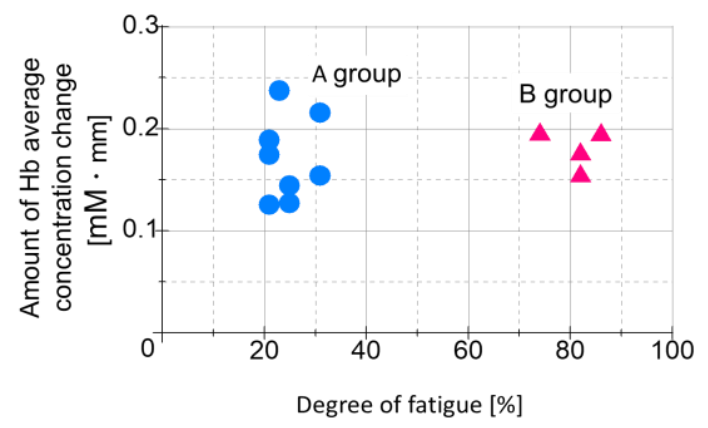

Fig. 8. Comparison of brain activity according to the degree of fatigue

\subsection{Relationship between the Degree of Fatigue and Mean Progress}

The degree of fatigue according to VAS and the mean progress (Figure 7) were weakly positively correlated (correlation coefficient 0.2). However, this correlation was not statistically significant $(\mathrm{P}>0.05)$.

We speculated that evaluating the degree of fatigue with respect to brain activity may reveal changes in of brain activity in relation to progress. Therefore, we classified the levels of fatigue into a low-fatigue group A and a highfatigue group B.

\subsection{Differences in Brain Activity Due to Fatigue}

Following the results of the relationship of fatigue with progress, we investigated the relationship between category of fatigue (low or high) and the change in oxy-Hb concentration (Figure 8). No evident changes in brain activity were observed during composition with regard to the degree of fatigue.

\section{Conclusions}

The present study reveals that brain activity is induced by the type of musical phrase that is being composed, the specific cognitive task, and the amount of motion being conducted while playing instruments. However, we did not identify an evident relationship between brain activity and progress during composition. The mean change in $\mathrm{Hb}$ concentration was analyzed; however, further analysis using a narrower range should be conducted to clarify whether the lack of correlation is valid. For instance, we have previously conducted experiments in which subjects were instructed to come up with a good idea, following which the activated channel is identified and analyzed. Another approach could be to analyze the instantaneous change in cerebral blood flow 
when subjects experience an inspiration. We collected data during the composition of four pieces of identical genre. However, this amount of data may be insufficient for comprehensive analyses. Further investigations should be conducted involving music composition of various genres, with increased numbers of subjects, thereby providing varied and sufficient data.

\section{Acknowledgment}

A part of this study was supported by a grant from Nagaoka University of Technology for collaborative research with National Institute of Technology.

\section{References}

(1) Yusuke Mitani, Takuya Kubo, Yuya Chiba, Yoshiko Maruyama, Moriya Kenji, Masahiro, Nakagawa : "Brain Activity During Listening to And Imagining Music:Does Imagining Music Provide a Similar Effect as Listening to Music?", Journal of the Institute of Industrial Applications Engineers, Vol.7, No.4, pp.127131,2019

(2) K. Moriya, I. Kurimoto, N. Ezaki, M. Nakagawa : "Influences of Listening to Music in Study Break on Brain Activity and Parasympathetic Nervous System Activity", Journal of the Institute of Industrial Applications Engineers, Vol.6, No.1, pp. 34-38, 2018

(3) Madoka Shimo, Keiko Sugo, Sachiko Agehara, Katsuo Sugita, Takuro Ishii, Masakazu Iwasaka : "Evaluation of the Level of "Music Relaxation" Utilizing Hemodynamics by Near-infra-red-spectroscopy", Bulletin of the Faculty of Education, Chiba University, Vol. 56, pp. 343-348, 2008

(4) Keisuke Teranishi and Hiroshi Hagiwara : "Characteristic Changes in the Brain Measured by NearInfrared Spectroscopy (NIRS) during "Aha" Experiences", J. Mobile Interactions, Vol. 1, No. 1, pp. 41-46, 2011

(5) Shinya Matsutani, Takamichi Taniguchi, Daisuke Hirano, Takashi Fujioka, Motoko Sugihara : "Effects of the Creativity of Activity on Prefrontal Cortex Activation: Measurements with Functional NearInfrared Spectroscopy (fNIRS)", Journal of the International University of Health and Welfare, Vol. 18, No. 2, pp. 50-57, 2013

(6) Kenta Hirayama, Keiichi Watanuki, Kaede Kazunori : "Brain Activation Analysis of Voluntary Movement and
Passive Movement Using Near-Infrared Spectroscopy", Transactions of the Japan society of mechanical engineers. C, Vol. 78, No. 795, pp. 3803-3811, 2012

(7) Takaki Shimura : "Prefrontal Lobe Measurement Using Near Infrared Spectroscopy -Evaluation of Early Detection Methods and Rehabilitation Methods of Dementia-", CORONA PUBLISHING CO., LTD, pp. $57-67,2009$ 